American Journal of Biochemistry and Biotechnology 1 (2): 103-106, 2005

ISSN 1553-3468

(C) 2005 Science Publications

\title{
Use of Cross-linking to Assess Subunit Interaction of Recombinant Human Coproporphyrinogen Oxidase
}

\author{
Jason R. Stephenson, Nancy E. Thomas, Jon A. Friesen and Marjorie A. Jones \\ Department of Chemistry, Illinois State University, Normal, IL 61790-4160
}

\begin{abstract}
To provide further evidence for a dimeric form of coproporphyrinogen oxidase reported using the conventional hydrodynamic methods, bifunctional cross-linkers were incubated with purified, recombinant human coproporphyrinogen oxidase to determine subunit interaction in solution. The use of cross-linkers provides an effective way to demonstrate subunit association and allows for assessment of activity upon covalent cross-linking. Following incubation with selected cross-linkers, enzyme apparent molecular weight was evaluated using SDS-PAGE and enzymatic activity was monitored by spectroscopy following HPLC. The predominate multimeric form of coproporphyrinogen oxidase observed had a mass that corresponded to a dimer, indicating that coproporphyrinogen oxidase most likely functions as a homodimer in solution.
\end{abstract}

Key words: Coproporphyrinogen oxidase, cross-linking, multimeric, protein structure

\section{INTRODUCTION}

Coproporphyrinogen oxidase (copro'gen oxidase [E.C. 1.3.3.3]) is the sixth enzyme in the heme biosynthetic pathway ${ }^{[1]}$. Porphyrins such as heme perform a variety of biological functions, especially as prosthetic groups of proteins necessary for transport of oxygen and oxidation/reduction reactions. Copro'gen oxidase catalyzes the conversion of coproporphyrinogen III (C-III) to protoporphyrinogen IX, a divinyl product, through the monovinyl intermediate harderoporphyrinogen. Current literature supports a dimeric active form of mammalian copro'gen oxidase, although there have been some discrepancies in the past. Batlle et al. ${ }^{[2]}$ purified copro'gen oxidase from rat liver and determined it to be an $80 \mathrm{kDa}$ monomer. Copro'gen oxidase purified from $S$. cerevisiae was shown to be a homodimer by Camadro et al. ${ }^{[3]}$, whereas Poulson and Polglase ${ }^{[4]}$ determined the protein from the same species to be a monomer. Bogard et al. ${ }^{[5]}$ purified copro'gen oxidase from mouse liver and reported a $70 \mathrm{kDa}$ homodimer. Yoshinaga and $\mathrm{Sano}^{[6]}$ purified copro'gen oxidase from bovine liver and determined the molecular structure to be a $71.6 \mathrm{kDa}$ monomer, whereas Kohno et al. ${ }^{[7]}$ reported the protein from bovine liver to be a homodimer, with an apparent molecular weight of 74 $\mathrm{kDa}$. Medlock and Dailey ${ }^{[8]}$ purified the recombinant human form of copro'gen oxidase and found it to have an apparent molecular weight of $37.5 \mathrm{kDa}$ by SDSPAGE. Martasek et al. ${ }^{[9]}$ also purified the recombinant human form and determined active copro'gen oxidase to have a molecular mass of $76 \mathrm{kDa}$, supporting a dimeric form. They also noticed that $5-10 \%$ of the total activity was found with a mass of $150 \mathrm{kDa}$, suggesting an additional tetramer form of copro'gen oxidase. The structure of oxygen-dependant copro'gen oxidase from Saccharomyces cerevisiae was recently evaluated by $\mathrm{x}-$ ray crystallography and shown to be a homodimer ${ }^{[10]}$. A summary of these literature sources is found in Table 1. Since these studies of the dimeric copro'gen oxidase native form were all determined using hydrodynamic procedures, we wished to extend these studies. Therefore, we carried out experiments using selected protein cross-linkers to determine subunit interaction in solution. The assessment of active enzyme in solution has not yet been evaluated by use of protein covalent cross-linkers. These cross-linkers have the advantage of good functional group specificity and controlled size so that only selected residues within known distances will be cross-linked. Thus, these studies should provide strong support for the current evidence of copro'gen oxidase assuming a native dimer form in solution. In addition, due to the selectivity of the cross-linkers, we can probe the abundance of lysine or cysteine residues at the subunit interface. We now report both the apparent molecular weight and the enzymatic activity of copro'gen oxidase with and without incubation with selected cross-linkers.

\section{MATERIALS AND METHODS}

Two bifunctional cross-linker molecules, $\mathrm{BS}^{3}$ [bis(sulfosuccinimidyl) suberate] and Sulfo-SMCC [sulfosuccinimidyl 4-( $N$-maleimidomethyl) cyclohexane-1-carboxylate] (purchased from Pierce), were used in these studies. $\mathrm{BS}^{3}$ forms covalent adducts with primary amines of a protein that are within a distance of $11.4 \AA$. Sulfo-SMCC forms covalent adducts with primary amines and sulfhydryl groups that

Corresponding Author: Marjorie A. Jones, Department of Chemistry, Illinois State University, Normal, IL 61790-4160.

Tel.: 1-309-438-2366; Fax: 1-309-438-5538 
are within a distance $11.6 \AA$. Following incubation of highly purified, cloned human enzyme with either cross-linker, with or without substrate, the reactions were evaluated by sodium dodecyl sulfate polyacrylamide gel electrophoresis (SDS-PAGE) and assessment of enzymatic activity was performed.

Cultures of $E$. coli expressing 6x-his-tagged human cloned copro'gen oxidase were grown at $37^{\circ} \mathrm{C}$; cells were then harvested by centrifugation at $4420 \mathrm{xg}$ for 5 minutes. The $E$. coli cells were lysed using a French Press cell at 20,000 psi followed by centrifugation at $11,300 \mathrm{xg}$ for 10 minutes. The supernatant was then applied to a nickel affinity column (Qiagen) in order to bind the 6x-his-tagged copro'gen oxidase, which was eluted with $250 \mathrm{mM}$ imidazole following the method of Medlock and Dailey ${ }^{[6]}$. BS $^{3}$ and Sulfo-SMCC were freshly prepared in dimethyl sulfoxide for each experiment in stock solutions of either $100 \mathrm{mM}$ or 250 $\mathrm{mM}$. Following pre-incubation with $10 \mu \mathrm{L}$ of crosslinkers, at final concentrations of $9 \mathrm{mM}$ or $23 \mathrm{mM}$, for 30 minutes at room temperature, an enzyme assay using $0.05 \mathrm{mg}$ of protein $\mathrm{mL}^{-1[11]}$ and SDS-PAGE using 0.62 $\mathrm{mg}$ of protein $\mathrm{mL}^{-1}{ }^{112]}$ were then performed plus or minus addition of coproporphyrinogen III as substrate. Incubation of enzyme without cross-linkers, with DMSO and incubation of substrate with cross-linker and no enzyme were used as controls. Neither crosslinker affected the substrate and DMSO did not perturb the enzyme. The enzymatic and SDS-PAGE reactions were carried out in a total volume of $310 \mu \mathrm{L}$ and $110 \mu \mathrm{L}$, respectively.

\section{RESULTS AND DISCUSSION}

Changes in enzyme apparent molecular weights were determined by SDS-PAGE, using 12\% acrylamide. Relative to molecular weight standards, copro'gen oxidase, absent of incubation with crosslinker, had an apparent molecular weight of $37.5 \mathrm{kDa}$ (Fig. 1). SDS-PAGE analysis of human-cloned copro'gen oxidase incubated with either cross-linker indicated 3 major higher molecular weight bands (approximately $65 \mathrm{kDa}, 86 \mathrm{kDa}$ and $107 \mathrm{kDa}$ ) relative to enzyme not incubated with selected cross-linkers. The data clearly indicate that dimerization and other higher molecular weight complexes could be detected following addition of $\mathrm{BS}^{3}$. Cross-linking with SulfoSMCC was not as efficient as with $\mathrm{BS}^{3}$, which is most likely due to fewer cysteine residues within the proper distance of lysines to form covalent adducts at the dimer interface. In addition to the higher molecular weight bands observed, one form of copro'gen oxidase in the presence of either cross-linker, at a final concentration of $9 \mathrm{mM}$, had an apparent monomer molecular weight of approximately $40 \mathrm{kDa}$. This increase in apparent molecular weight and the marked band width broadening clearly implies intramolecular cross-linking. Intramolecular covalent adducts will

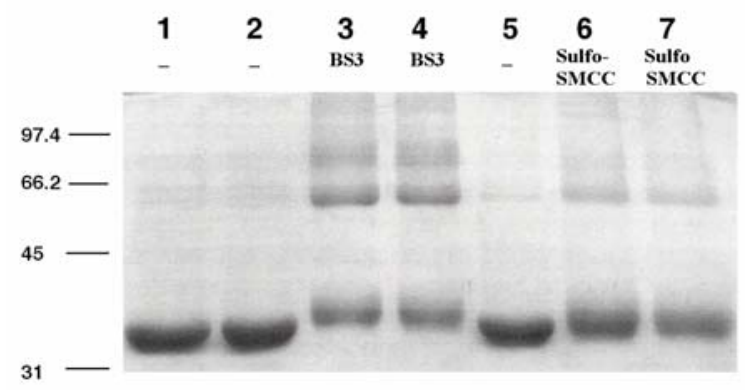

Fig. 1:SDS-PAGE of Copro'gen oxidase. Representative gel from SDS-PAGE of copro'gen oxidase with and without crosslinkers. Molecular weight standards $(\mathrm{kDa})$ are indicated at the left. Lanes 1, 2 and 5, copro'gen oxidase without cross-linkers. Lanes 3 and 4 , copro'gen oxidase incubated with $9 \mathrm{mM} \mathrm{BS}^{3}$, with and without substrate, respectively; lanes 6 and 7, copro'gen oxidase incubated with $9 \mathrm{mM}$ Sulfo-SMCC with and without substrate respectively. An enzyme concentration of 0.62 $\mathrm{mg} \mathrm{mL}{ }^{-1}$ was used in these incubations so that 10 $\mu \mathrm{g}$ of protein was loaded per lane on SDS-PAGE

have an effect on the migration pattern of proteins on SDS-PAGE since the proteins can no longer denature completely. The higher apparent molecular weight bands migrating at 65,86 and $107 \mathrm{kDa}$ clearly imply that dimeric, trimeric and tetrameric complexes are being covalently joined by the cross-linker. The apparent molecular weights of these complexes are different from expected molecular weight calculations from known copro'gen oxidase monomer and dimer $\mathrm{kDa}$ weights. This can be explained by the retention of the globular structure of cross-linked subunits relative to the linear, random coil of a completely denatured protein in the presence of the SDS detergent. Thus, the assumption of all proteins running as a function of molecular weight on an SDS-PAGE is not supported when the cross-linkers are used. Therefore, the apparent molecular weights of the higher molecular weight complexes being formed are not assumed to be accurate, but the observation of these bands does support multiple subunit interactions of copro'gen oxidase. Thus the formation of higher molecular weight complexes upon covalent cross-linking in solution reported here supports the current acceptance of a dimeric native structure of copro'gen oxidase. These data also support the $\mathrm{x}$-ray crystallographic structure data indicating dimerization of copro'gen oxidase can occur in solution ${ }^{[10]}$. These data also correlate with the report of Medlock and Dailey that human-cloned copro'gen oxidase has an apparent monomeric molecular weight of $37.5 \mathrm{kDa}$ by SDSPAGE. The findings of Martasek et al. ${ }^{[9]}$ showing an active tetramer form of copro'gen oxidase are supported by the apparent molecular weight bands migrating as 86 and $107 \mathrm{kDa}$, indicating that in 
Am. J. Biochem. \& Biotechnol. 1 (2): 103-106, 2005

\begin{tabular}{|c|c|c|c|c|}
\hline Species & Reported Molecular Weight & Reported Form & Technique Used & Reference \\
\hline Rat liver & $80 \mathrm{kDa}$ & Monomer & Sephadex Gel Filtration & Batlle et al. ${ }^{[2]}$ \\
\hline S. cerevisiae & $\begin{array}{l}\sim 35 \mathrm{kDa}-\text { Monomer } \\
\sim 70-75 \mathrm{kDa}-\text { Dimer }\end{array}$ & Homodimer & X-ray Crystallography & Philips et al. ${ }^{[10]}$ \\
\hline & $75 \mathrm{kDa}$ & Monomer & $\begin{array}{l}\text { Ultrogel AcA } 44 \text { Gel Filtration } \\
\text { Sephadex Gel Filtration }\end{array}$ & $\begin{array}{l}\text { Camadro et al. }{ }^{[3]} \\
\text { Poulson and Polglase }{ }^{[4]}\end{array}$ \\
\hline Mouse Liver & $\begin{array}{l}35 \mathrm{kDa}-\text { Monomer } \\
70 \mathrm{kDa}-\text { Dimer }\end{array}$ & Homodimer & Ultrogel AcA 44 Gel Filtration & Bogard et al. ${ }^{[5]}$ \\
\hline Bovine Liver & $\begin{array}{l}71.6 \mathrm{kDa} \\
37 \mathrm{kDa}-\text { Monomer } \\
74 \mathrm{kDa}-\text { Dimer }\end{array}$ & $\begin{array}{l}\text { Monomer } \\
\text { Homodimer }\end{array}$ & $\begin{array}{l}\text { Sephadex Gel Filtration } \\
\text { SDS-PAGE Gel Filtration }\end{array}$ & $\begin{array}{l}\text { Yoshinaga and Sano }{ }^{[6]} \\
\text { Kohno et al. } .^{[7]}\end{array}$ \\
\hline Human & $\begin{array}{l}37.5 \mathrm{kDa} \\
76 \mathrm{kDa} \text { - Dimer } \\
\text { 39.7-Monomer }\end{array}$ & Homodimer & $\begin{array}{l}\text { SDS-PAGE } \\
\text { Ultrogel AcA } 44 \text { Gel Filtration }\end{array}$ & $\begin{array}{l}\text { Medlock and Dailey }{ }^{[8]} \\
\text { Martasek et al. } .^{\left[{ }^{2}\right]}\end{array}$ \\
\hline
\end{tabular}

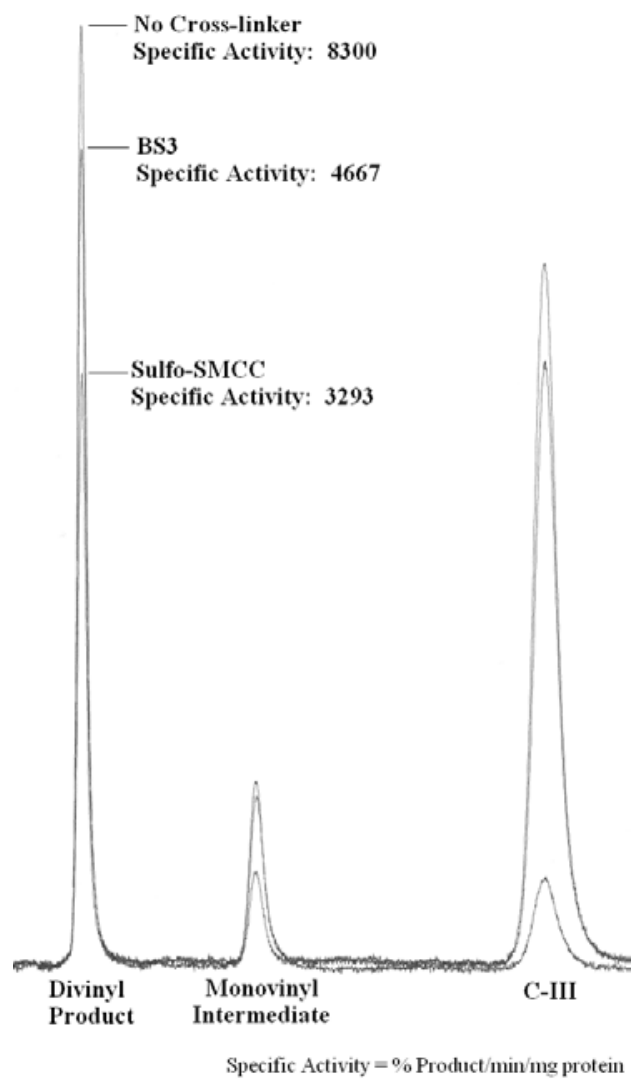

Fig. 2: Superimposed representative HPLC chromatograms following enzymatic assay. HPLC was performed on a Beckman normal phase $4.6 \mathrm{~mm} \times 25 \mathrm{~cm} 5 \mu$ silica column with a solvent of 35/65 (v/v) ethyl acetate/cyclohexane. Chromatograms show product formation and calculated specific activity (\% product $/ \mathrm{min} / \mathrm{mg}$ protein)

solution, the enzyme can retain activity in an oligomeric form. Incubation with the selected crosslinkers with and without substrate prior to SDS-PAGE showed no difference in band patterns, suggesting that substrate does not protect against covalent adduct formation and does not affect subunit interaction. The same cross-linking patterns were observed for either concentration of $\mathrm{BS}^{3}$ or Sulfo-SMCC.

The enzyme was active in the presence of either cross-linker, retaining approximately $50 \%$ of the original activity when using coproporphyrinogen III as substrate (Fig. 2). Copro'gen oxidase incubated without cross-linker had a specific activity (\% product $/ \mathrm{min} / \mathrm{mg}$ protein) of 8300 . Enzyme incubated with $\mathrm{BS}^{3}$ had a specific activity of 4667 , which is $56 \%$ of the activity of enzyme not cross-linked. Copro'gen oxidase incubated with sulfo-SMCC had a specific activity of 3293, retaining $40 \%$ of original activity. This decrease in activity suggests that the active form of the enzyme is being compromised due to covalent adduct formation. At this time we cannot distinguish if this decrease in activity is due to effects on single amino acid residues or on subunit interactions. We speculate that there is a dynamic equilibrium between the different subunits of copro'gen oxidase and that these interactions have not yet been understood. Such interactions may have important implications for porphyric patients between and during the episodes of porphyria crises.

\section{ACKNOWLEDGMENTS}

This work was supported by an ISU University Research Grant (URG).

\section{REFERENCES}

1. Sano, S. and S. Granick, 1961. Mitochondrial coproporphyrinogen oxidase and protoporphyrin formation. J. Biol. Chem., 236: 1173-1180.

2. Batlle, A.M. del D., A. Benson and C. Rimington, 1965. Purification and properties of coproporphyrinogenase. Biochem. J., 97: 731-740.

3. Camadro, J.M., H. Chambon, J. Jolles and P. Labbe, 1986. Purification and properties of coproporphyrinogen oxidase from the yeast Saccharomyces cerevisiae. Eur. J. Biochem., 156: 579-587. 
4. Poulson, R. and J.W. Polglase, 1975. The enzymic conversion of protoporphyrinogen IX to protoporphyrin IX.. J. Biol. Chem., 250: 12691274.

5. Bogard, M., J.M. Camdro, Y. Nordmann and P. Labbe, 1989. Purification and properties of mouse liver coproporphyrinogen oxidase. Eur. J. Biochem., 181: 417-421.

6. Yoshinaga, T. and S. Sano, 1980. Coproporphyrinogen oxidase: Purification, properties and activation by phospholipids. J. Biol. Chem., 255: 4722-4726.

7. Kohno, H., T. Furukawa, T. Yoshinaga, R. Tokunaga and S. Taketani, 1993. Coproporphyrinogen oxidase: Purification, molecular cloning and induction of mRNA during erythroid differentiation. J. Biol. Chem., 268: 21359-21363.

8. Medlock, A.E. and H.A. Dailey, 1996. Human coproporphyrinogen oxidase is not a metalloprotein. J. Biol. Chem., 271: 32507-32510.
9. Martasek, P., J.M. Camadro, C.S. Raman, M.C. Lecomte, J.P. Le Caer, B. Demeler, B. Grandchamp and P. Labbe, 1997. Human coproporphyrinogen oxidase. Biochemical characterization of recombinant normal and R231W mutated enzymes expressed in E. coli as soluble, catalytically active homodimers. Cell. Mol. Biol., 43: 47-58.

10. Philips, J.D., F.G. Whitby, C.A. Warby, P. Labbe, C. Yang, J.W. Pflugrath, J.D. Ferrara, H.A. Robinson, J.P. Kushner and C.P. Hill, 2004. crystal structure of the oxygen-dependent coproporphyrinogen oxidase (Hem13p) of Saccharomyces cerevisiae. J. Biol. Chem., 279: 38960-38960.

11. Jones, M.A., P. Thientanavanich, M.D. Anderson and T.D. Lash, 2003. Comparison of two assay methods for activities of uroporphyrinogen decarboxylase and coproporphyrinogen oxidase. J. Biochem. Biophys., 55: 241-249.

12. Laemmli, U.K., 1970. Cleavage of structural proteins during assembly of the head of bacteriophage T4. Nature, 227: 680-685. 